\title{
Subnanosecond Thomson scattering setup for space and time resolved measurements with reduced background signal
}

\section{Citation for published version (APA):}

Kieft, E. R., Groothuis, C. H. J. M., Mullen, van der, J. J. A. M., \& Banine, V. Y. (2005). Subnanosecond Thomson scattering setup for space and time resolved measurements with reduced background signal. Review of Scientific Instruments, 76(9), 093503-1/6. https://doi.org/10.1063/1.2038667

DOI:

10.1063/1.2038667

Document status and date:

Published: 01/01/2005

\section{Document Version:}

Publisher's PDF, also known as Version of Record (includes final page, issue and volume numbers)

\section{Please check the document version of this publication:}

- A submitted manuscript is the version of the article upon submission and before peer-review. There can be important differences between the submitted version and the official published version of record. People interested in the research are advised to contact the author for the final version of the publication, or visit the $\mathrm{DOI}$ to the publisher's website.

- The final author version and the galley proof are versions of the publication after peer review.

- The final published version features the final layout of the paper including the volume, issue and page numbers.

Link to publication

\section{General rights}

Copyright and moral rights for the publications made accessible in the public portal are retained by the authors and/or other copyright owners and it is a condition of accessing publications that users recognise and abide by the legal requirements associated with these rights.

- Users may download and print one copy of any publication from the public portal for the purpose of private study or research.

- You may not further distribute the material or use it for any profit-making activity or commercial gain

- You may freely distribute the URL identifying the publication in the public portal.

If the publication is distributed under the terms of Article 25fa of the Dutch Copyright Act, indicated by the "Taverne" license above, please follow below link for the End User Agreement:

www.tue.nl/taverne

Take down policy

If you believe that this document breaches copyright please contact us at:

openaccess@tue.nl

providing details and we will investigate your claim. 


\title{
Subnanosecond Thomson scattering setup for space and time resolved measurements with reduced background signal
}

\author{
E. R. Kieft, C. H. J. M. Groothuis, and J. J. A. M. van der Mullen ${ }^{\text {a) }}$ \\ Department of Applied Physics, Eindhoven University of Technology, P.O. Box 513, 5600 MB Eindhoven, \\ The Netherlands \\ V. Banine \\ ASML Netherlands B.V., De Run 6501, 5504 DR Veldhoven, The Netherlands
}

(Received 23 May 2005; accepted 28 July 2005; published online 2 September 2005)

In order to reduce the level of recorded background radiation in Thomson scattering (TS) experiments, we have designed and built a new setup for time and space resolved subnanosecond TS. Compared to our old setup, the new one is based on a faster camera, a laser with shorter pulse duration, and an optical delay line in the laser path. It has been characterized both by ray-trace simulations of the spectrograph and test experiments using Rayleigh and rotational Raman scattering on atmospheric pressure nitrogen gas. Although it was designed in the first place for experiments on a vacuum arc discharge in tin vapor for production of extreme ultraviolet light, our setup can also be applied to other plasmas that emit high levels of radiation or in which fast phenomena play a role. (C) 2005 American Institute of Physics. [DOI: 10.1063/1.2038667]

\section{INTRODUCTION}

Thomson scattering (TS) spectroscopy is a reliable technique to measure the density $n_{e}$ and temperature $T_{e}$ of the electron gas in a plasma simultaneously. In this technique, a laser pulse is fired through the plasma and the light scattered by free electrons is recorded as a function of wavelength. It is often applied to plasmas that are located far from any vessel walls, such as plasmas for future nuclear fusion reactors. In such cases, scattering from atoms and ions, stray light reflections of the laser light, and light emission of the plasma itself are often not a problem for the measurement of the Thomson scattered spectrum.

However, in plasmas with a very low ionization degree, the wings of the Rayleigh scattered light from atoms competes with the TS spectrum. Further, in many types of "technological" plasmas, such as deposition plasmas or lamps, the electrodes and the walls can be very close to the regions of interest in the plasma, and a certain amount of reflected laser light from solid surfaces cannot be avoided. In these cases, a notch filter is needed to block the central wavelength in the scattered light spectrum. In the past, an atomic absorption cell has been applied for this purpose. ${ }^{1}$ Another method is the application of a so-called triple grating spectrograph (TGS). ${ }^{2,3}$ In our group, a TS setup including a TGS has been designed and built by Marco van de Sande. ${ }^{3}$ It has been applied to a number of different plasma types; see, e.g., Refs. 3-7.

Another problem that can occur in determining the shape of the TS spectrum from recorded images is the competition of the TS signal with background radiation that is emitted by the plasma itself. In certain cases, the plasma radiation will

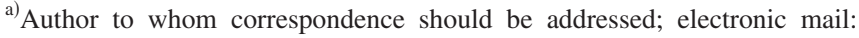
j.j.a.m.v.d.mullen@tue.nl
}

consist just of a limited number of spectral lines. In such cases, it can be sufficient to select a wavelength for Thomson scattering at which the background spectrum is relatively clean, for example by application of a wavelength-tunable laser. However, if the plasma radiation forms a (quasi) continuum over a large wavelength range, simply selecting a suitable laser wavelength or blocking specific spectral lines is not enough to solve the problem. Instead, a more general method needs to be applied to improve the signal to background ratio.

This was found to be true for the case of our vacuum arc discharge in tin vapor. Pulsed discharge plasmas in tin vapor are candidate sources of extreme ultraviolet (EUV) light for application in next generation lithography tools. Our vacuum arc discharge is an experimental EUV source that was designed and built at the Institute of Spetroscopy of the Russian Academy of Sciences. Due to its geometry, it has proven to be particularly accessible to different diagnostic techniques. ${ }^{8-10}$ In a previous series of experiments, we used the existing TS setup mentioned earlier, with some small modifications, to perform Thomson scattering on this plasma. ${ }^{10}$ Our TS experiments were successful in providing useful data for the so-called "prepinch phase" of the discharge, during which a strong electric current heats and ionizes the plasma at a high rate. Electron densities were found to increase from about $10^{23}$ to $10^{24} \mathrm{~m}^{-3}$, while the electron temperature increased from about 5 to over $30 \mathrm{eV}$.

Even higher electron densities of more than $10^{25} \mathrm{~m}^{-3}$ were expected for the pinch phase, while much lower densities could occur for parts of the so-called ignition and decay phases. However, in the same set of experiments it was found that it was not possible with the existing setup to do measurements for these other phases of the discharge. This is due to a too high level of background radiation emitted by the plasma in those phases. A background subtraction 
method was used to distinguish the Thomson scattered signal from the background, but random pulse-to-pulse fluctuations in the background prevented this method from working sufficiently well. In principle, longer experiments (i.e., integration over more discharge pulses) could be used to reduce the relative magnitude of the background fluctuations, but this approach is limited by long-term drifts in the behavior of the discharge, for example due to electrode erosion. Therefore, an alternative method to reduce the relative level of background radiation was needed. Such a reduction can, in principle, be achieved in two ways: the first is to acquire a certain TS signal from a smaller detection volume-that is, to use a narrower laser focus in combination with a narrower entrance slit for the spectrograph. The second is to record the signal in a shorter time.

In this article, we present the design and construction of a setup for space and time resolved subnanosecond (ns) Thomson scattering. It has been built as a flexible tool that can be applied to a range of different plasma types, but with the specific application mentioned earlier kept in mind. Both methods of background signal reduction have been pursued, with the main emphasis being on the latter.

The design of the new setup consists of two main parts. First, in Sec. II, the need for an optical delay line in the laser path is discussed, and the layout of the laser table is given. In Sec. III, the new triple grating spectrograph (TGS-II) is described, and we present the results of ray-trace simulations of this spectrograph. Finally, in Sec. IV, we describe the results of test experiments that we have performed to characterize the setup as a whole. In this article, we will frequently refer back to specifics of the old system to indicate which changes have been made.

The new setup has already been applied for actual experiments on the tin vapor discharge; the results of these measurements will be presented in a separate publication.

\section{LASER PATH}

\section{A. Synchronization of laser and camera}

A detailed description of the existing, previously used setup for TS can be found in Ref. 3 and the adjustments made for application to the tin vapor discharge are described in Ref. 10. In this old setup, a frequency doubled neodymium-doped yttrium aluminum garnet (Nd:YAG) laser pulse (at $532 \mathrm{~nm}$ ) of about $170 \mathrm{~mJ}$ pulse energy and $7 \mathrm{~ns}$ full width at half maximum (FWHM) duration was applied, and the camera gate time was set to $5 \mathrm{~ns}$ (with the camera delay being optimized to the maximum of the laser pulse intensity). Compared to this previously existing situation, a reduction of the time needed for a single measurement means, in the first place, that a shorter gate time has to be applied for the intensified charge-coupled device (ICCD) camera. In the new setup, we use a 4Picos camera from Stanford Computer Optics, which has a minimum specified gate time of 200 ps. To ensure sufficiently homogeneous illumination of the sensor by the intensifier, we applied a slightly longer gate width in practice.

To "fit" the laser pulse into the time window imposed by the camera gating, also a shorter laser pulse length had to be applied. In the new setup, the laser pulse is provided by the SL312 laser from Ekspla, Lithuania. Through stimulated Brillouin scattering, a Nd:YAG laser pulse is compressed from a few ns to $150 \mathrm{ps}$ duration. The maximum output of the laser at the second harmonic is about $120 \mathrm{~mJ}$ per pulse.

Finally, to ensure that the TS signal indeed arrives at the camera just when the camera is being gated, a proper synchronization of the laser pulse and camera gate is required. Commercially available laser systems that provide the desired laser wavelength, pulse duration, and pulse energy, can in general not be operated with sufficient accuracy in the time domain; also, electronic synchronization signals from the SL312 and other laser systems exhibit too much time jitter-on the order of $1 \mathrm{~ns}$ - to use them for triggering the camera. With such poor synchronization, the effective average Thomson signal recorded during the gating of the camera, would be strongly reduced compared to the signal at the peak of the laser pulse.

We solved this problem by asking the laser manufacturer to place a fast photodiode inside the laser head; a beam splitter, located in the laser path before the amplification stage, sends a small part of the pulse energy to the photodiode, which converts it into a highly stable electric signal that can be used to trigger the camera. This approach has one obvious drawback: the trigger signal for the camera is only generated when the laser pulse has already been formed. It takes the signal some $15 \mathrm{~ns}$ to travel from the laser to the camera and the camera takes at least another $65 \mathrm{~ns}$ to gate its intensifier after arrival of the trigger signal. Therefore, the TS light should arrive at the camera at least $80 \mathrm{~ns}$ after the generation of the trigger signal.

In the old design, the optical path, from the laser head to the plasma and from the plasma to the camera entrance, was only about $10 \mathrm{~m}$ long, which corresponds to about $33 \mathrm{~ns}$ delay. Therefore, for the new setup, a dedicated delay line in the optical path for the laser pulse was needed to provide the additional delay.

A schematic overview of the design of the sub-ns TS system is shown in Fig. 1. The two main parts, apart from the plasma chamber itself, are the laser table and the triple grating spectrograph. The latter is discussed in Sec. III; first we focus on the delay line.

\section{B. Optical delay line}

The first requirement for the delay line can be derived from the numbers quoted earlier: a sufficiently long additional delay of at least $50 \mathrm{~ns}$ has to be generated; this corresponds to a path length of about $15 \mathrm{~m}$. However, equally important requirements are good efficiency (so that sufficient laser energy can be focused into the plasma), ease and stability of alignment, and resistance against high laser powers.

This combination of requirements was met by making use of solid-glass corner cubes (CCs). These have the special property that any incident light ray (within a certain solid angle) gets reflected in the exactly parallel, opposite direction. The principle of using corner cubes for multipass optical cells was discussed by Vitushkin. ${ }^{11}$ Klövekorn et al. ${ }^{12}$ used a CC in a variable-length optical delay line that is independent of the mechanical quality of the translation stage. 


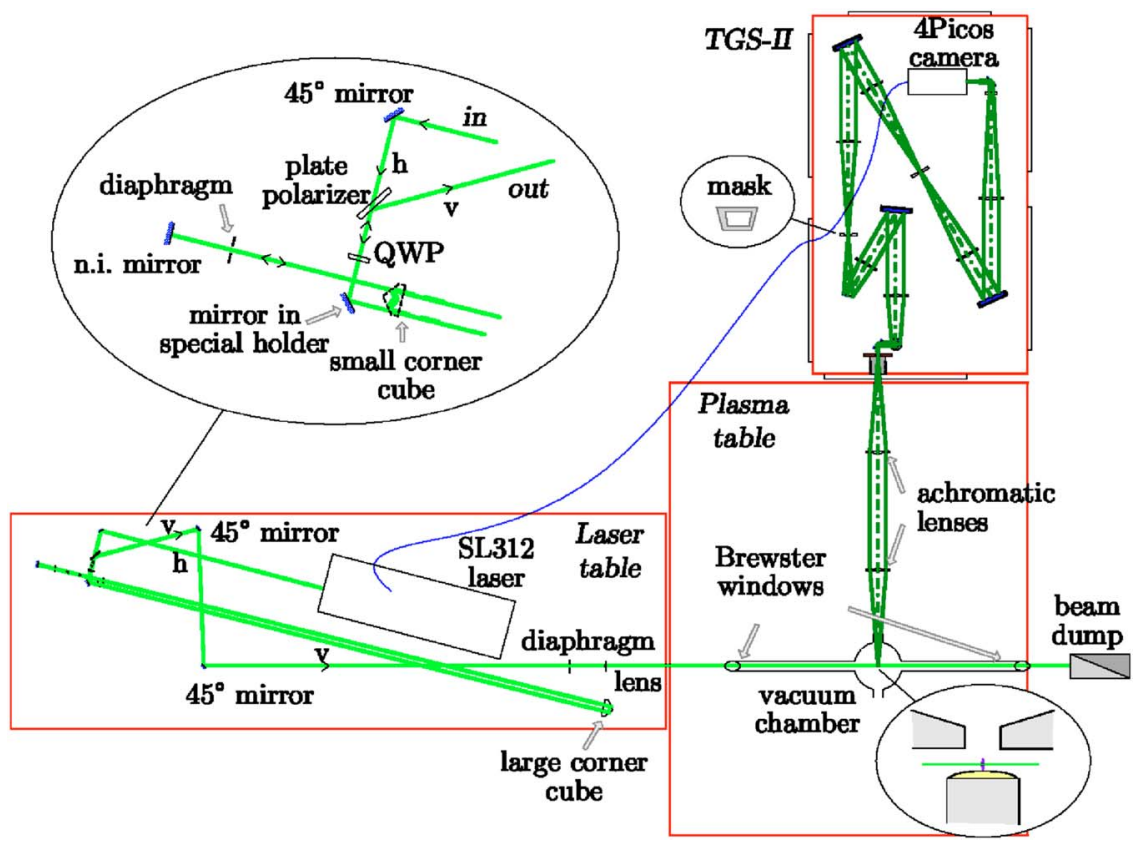

FIG. 1. Schematic overview of the complete TS setup, with the laser table shown on the left-hand side (see the top left for an enlargement of the leftmost part), and the TGS-II spectrograph at the top right. After passing through the plasma inside the vacuum chamber, the laser beam is collected by a beam dump. The scattered light is collected and imaged onto the entrance slit of the spectrograph by two achromatic lenses, of which the opening angle is matched to that of the spectrograph.

In our setup, we use two CCs of unequal sizes. They are arranged to have parallel optical axes, with the axis of the largest one being directly above that of the smallest. The path of the laser beam is depicted in Fig. 2. A $45^{\circ}$ mirror first directs the laser beam towards the top half of the large CC. The reflected beam is shifted both horizontally and vertically, and is directed towards the small CC. The beam is reflected and shifted horizontally by the small $\mathrm{CC}$ and returns to the large CC. After another reflection by the large CC, the beam passes over the top of the small CC and along the side of the $45^{\circ}$ mirror, and reaches a normal-incidence (n.i.) mirror. From the n.i. mirror, the laser beam is reflected over exactly the same path; hence, it travels the distance between both CCs for a total of eight times, and exits the delay line in the same position and opposite direction it entered it in.

This delay line provides a total path length of about $18 \mathrm{~m}$, which is limited just by the size of the table. This is more than the requirement derived above; however, any excess delay of the TS signal pulse can be compensated for by setting an appropriate delay in the internal delay generator of the ICCD camera.

The laser pulse is guided from the laser head to the delay line via a combination of a plate polarizer and a quarter wave plate. Initially, the pulse is horizontally polarized and gets transmitted by the polarizer, which is positioned at the Brewster angle with respect to the laser beam. After two passes through the quarter wave plate (one before and one after passing through the delay line) it has become vertically polarized, and it gets completely reflected by the polarizer. This way, both beams are separated, and next the returning beam is sent to the plasma chamber. The complete delay line is shown at the bottom left of Fig. 1; an enlargement of part of the delay line, including the entrance and exit, can be found at the top left.

The laser beam is focused into the plasma by a lens with $1 \mathrm{~m}$ focal length; the vertically polarized beam crosses the plasma in the horizontal direction. In the case of the tin vapor discharge setup, it was guided into and out of the vacuum chamber using Brewster angle windows, to minimize energy losses and production of stray light.

\section{SPECTROGRAPH}

\section{A. Principle of the triple grating spectrograph}

Laser light that is Thomson scattered from the plasma in the perpendicular horizontal direction is collected by two achromatic lenses and focused onto the horizontal entrance slit of a triple grating spectrograph, the TGS-II. Different positions along the entrance slit correspond to different positions along the plasma-laser beam intersection. The design of the TGS-II is based on that of the original TGS by Van de Sande. ${ }^{3}$ In this design, three spectrographs are placed in series, of which the first two are arranged in a subtractive configuration. A mask, placed in the image plane of the first spectrograph, blocks the central wavelength. Next, the second spectrograph folds the spectrum back into a single line, so that the light can pass through an intermediate slit between the second and third spectrographs. Any light at the laser wavelength that was able to pass the mask due to imaging imperfections and stray reflections inside the TGS, is blocked by the intermediate slit. This arrangement turns the first two spectrographs into a strong stray light filter. Finally, the third spectrograph, behind the intermediate slit, provides the spectral dispersion needed to record the spatially resolved spectrum on the ICCD camera. An image rotator, con- 


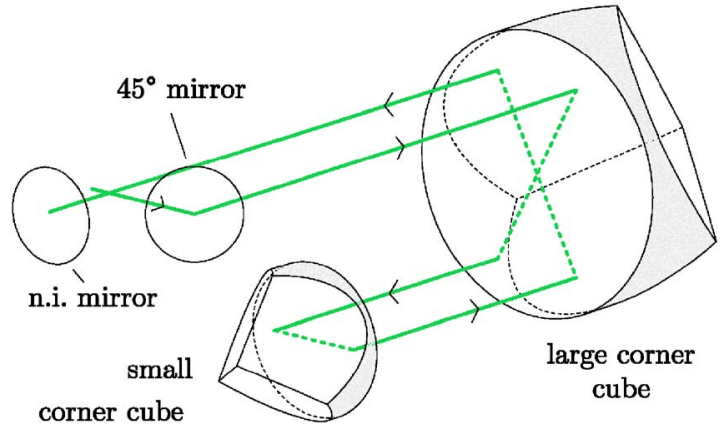

FIG. 2. Schematic drawing of the laser path between the corner cubes in the optical delay line. The parts of the optical path inside the corner cubes have been omitted for clarity. Distances between the optical elements are not to scale; in particular, in the actual setup, the large corner cube is placed much further away from the other elements, to obtain a long path length.

sisting of three mirrors, is located just behind the entrance slit of the TGS, so that in the camera image, the wavelength axis is in the horizontal direction, while the vertical direction corresponds to the spatial dimension. The changes to the original design that have been made in the new one-as schematically depicted in Fig. 1-are discussed next.

\section{B. Design of the TGS-II}

In the new design, first of all, we use different gratings, with only 600 instead of 1800 grooves per millimeter. This is needed to expand the spectral range of the spectrograph from about 20 to $50 \mathrm{~nm}$. Dependent on the orientation of the gratings, we can now record both wings of the TS spectrum symmetrically up to $25 \mathrm{~nm}$ away from the central wavelength, or select just one side of the spectrum and record it up to $50 \mathrm{~nm}$ away from the laser wavelength. The former method makes fitting of the spectrum to a theoretical curve easier, as information from both sides of the spectrum is available; however, the latter is necessary to do measurements on the highest density parts of the tin vapor discharge, for which the TS spectrum is strongly collective. In this case, the "notch mask" that blocks just the central wavelength, is replaced by an "edge mask," since only one half of the spectrum is recorded by the camera.

Further, the focal lengths of all lenses but the last were reduced from 600 to $400 \mathrm{~mm}$. This allowed us to apply smaller diameter lenses while maintaining a large collection angle. A larger focal length of $600 \mathrm{~mm}$ was used for the last lens to magnify the image 1.5 times and thus obtain better spatial resolution. Also, an extra mirror was placed just before the camera to fold the optical path, and be able to fit the design onto a somewhat smaller table than the original one.

A narrower entrance slit, of only 100 instead of $250 \mu \mathrm{m}$ width was applied for two reasons: first, in combination with a narrower laser focus, it helps to improve the signal-tobackground ratio. Second, it partly compensates for the reduction of spectral resolution caused by the new set of gratings.

Finally, in our design, certain translation stages for optical elements that were present in the old design, were left out to improve the stability of the alignment against positional drift. Such drifts during a single experiment, or between ex- (a)

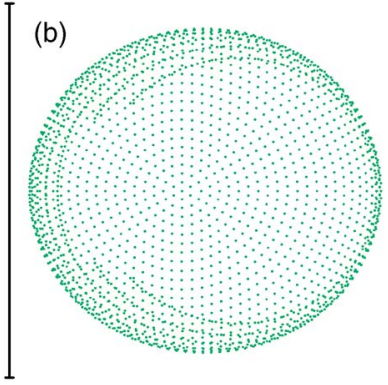

(c)
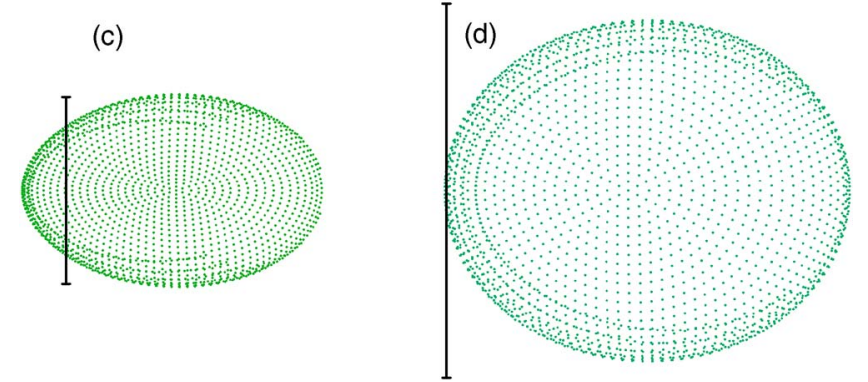

FIG. 3. Ray-trace simulated image spots in ZEMAX for four different cases: (a) $x=0 \mathrm{~mm}, \Delta \lambda=0 \mathrm{~nm}$; (b) $x=0 \mathrm{~mm}, \Delta \lambda=-25 \mathrm{~nm}$; (c) $x=5 \mathrm{~mm}, \Delta \lambda$ $=0 \mathrm{~nm}$; and (d) $x=5 \mathrm{~mm}, \Delta \lambda=-25 \mathrm{~nm}$, for a central wavelength of $532 \mathrm{~nm}$. Here, $x$ and $\Delta \lambda$ represent shifts from the center of the image in the spatial and wavelength directions, respectively. The vertical bars represent sizes of $100 \mu \mathrm{m}$ for parts (a) and (c), and $200 \mu \mathrm{m}$ for parts (b) and (d).

periments, can seriously reduce the efficiency of the spectrograph, and make frequent realignment of the system necessary. Instead, in certain cases we accepted a small deviation from the "ideal" optical path for certain optical elements. Such deviations can be compensated for by similar, but opposite shifts of other elements (which do still have the required degree of freedom of alignment), without seriously affecting the overall resolution of the system (see later). Specifically, of each set of two lenses, only one had a translation stage in the horizontal direction perpendicular to the optical axis. For the first spectrograph, the horizontal positioning of the focus was done by adjustment of the first folding mirror, which is located after the second lens. Further, we required a precise translation stage in the longitudinal direction for only one of each pair of lenses (with the other just being placed on a carrier on an optical rail).

\section{Ray-trace simulations}

The ZEMAX optical design program was used to do ray trace simulations of the TGS-II, including the two lenses in front of it. In this way, we have been able to evaluate its spatial and spectral resolution. Calculations have been performed for multiple spatial positions inside the detection volume in the plasma, and for multiple wavelengths. Examples of image spots in the camera plane are depicted in Fig. 3. Table I summarizes the results.

The same program has been used to test the influence of possible misalignments, such as discussed earlier, on the final resolution. The following cases have been tested: (a) a rotation of the third grating by $0.2 \mathrm{deg}$ in the horizontal plane; (b) a horizontal shift of $1 \mathrm{~mm}$ perpendicular to the optical axis of the first lens of the last spectrograph; (c) a similar shift of the first lens of the second spectrograph, com- 
TABLE I. Root mean squares $r_{\text {RMS }}$ of the distances of individual ray-trace points from each spot center, produced by ZEMAX for different horizontal positions inside the plasma and different wavelengths. $x$ and $\Delta \lambda$ are defined as the shifts from the central position and the central wavelength, respectively, which is $532 \mathrm{~nm}$ in this case. For evenly distributed points in a round image spot, the spot radius $R$ equals $\sqrt{2} r_{\text {RMS }}$.

\begin{tabular}{lrrrrrrrrrr}
\hline \hline$x(\mathrm{~mm})$ & -5.0 & -2.5 & 0.0 & 0.0 & 0.0 & 0.0 & 0.0 & 2.5 & 5.0 & 5.0 \\
$\Delta \lambda(\mathrm{nm})$ & 0.0 & 0.0 & -25.0 & -12.5 & 0.0 & 12.5 & 25.0 & 0.0 & 0.0 & -25.0 \\
\hline$r_{\text {RMS }}(\mu \mathrm{m})$ & 52.9 & 16.7 & 75.3 & 35.3 & 7.3 & 21.6 & 33.4 & 16.8 & 53.3 & 83.2 \\
\hline \hline
\end{tabular}

pensated for by an appropriate shift of the second lens of the same spectrograph; and (d) a similar shift of the first lens of the first spectrograph, compensated for by a shift of the first folding mirror, and a longitudinal shift of the second lens (to keep the optical path length between that lens and the mask constant). In cases (a) and (b) no compensation by a shift of another element is necessary since these would just lead to a small shift of the image on the camera, which could easily be digitally compensated for during data processing. Therefore, we included compensating shifts of other elements only for cases (c) and (d).

For all tested cases, we found that the alignment deviations have little or no influence on the resolution of the spectrograph.

To evaluate the overall spatial and spectral resolution of the system, the numbers given in Table I should be compared to the width of the image of the entrance slit on the camera, and the width of the apparatus profile of the camera intensifier itself. These are $164 \mu \mathrm{m}$ and on the order of $100 \mu \mathrm{m}$, respectively. For the points near the center of the image, these numbers are limiting the overall resolution; at the lower wavelength limit, and at the outsides of the spatial profile, the smallest resolvable features according to Table I are somewhat larger, up to just over $200 \mu \mathrm{m}$ in diameter. However, in most experiments, only measurements near the spatial center of the image, $x=0 \mathrm{~mm}$, have been done. Therefore, it can be concluded that ideally, the spatial and spectral resolution of the system - taking into account the geometrical magnification of the system, and the dispersion caused by the gratings—are about $70 \mu \mathrm{m}$ and $0.4 \mathrm{~nm}$, respectively.

\section{CHARACTERIZATION}

Test experiments have been performed to determine the efficiency of the delay line and the spectrograph, and to check that their combination works properly.

First, we tested the delay line efficiency by simply comparing laser power measurements just behind the laser exit and at the end of the delay line, just before the beam is focused into the plasma chamber. We have measured an efficiency of $78 \%$ for the fully optimized alignment of the laser table. We found that, in practice, only a quick optimization of the orientation of both mirrors inside the delay line itself is needed to have it function properly; therefore, day-to-day optimization is just a matter of seconds. On the other hand, a full optimization of the efficiency of the optical path requires a bit more work. In this case, the angles to the optical axis of the $45^{\circ}$ mirrors and the plate polarizer need to be as close as possible to their nominal values to minimize transmission and reflection losses, and the orientation of the quarter wave plate needs to be optimized to ensure that the returning beam from the delay line is perfectly vertically polarized.

Although the maximum output from the laser was about $120 \mathrm{~mJ}$ per pulse, in our actual experiments, we limited the laser pulse energy inside the plasma to about $50 \mathrm{~mJ}$, mainly to limit disturbance of the plasma. It should be noted, however, that already at this setting, we found ourselves close to the limit of laser power that the delay line could handle. We found evidence of surface damage on the large corner cube at an only slightly higher laser energy.

To test the triggering of the camera, we performed Rayleigh scattering measurements on atmospheric pressure nitrogen gas. Naturally, the mask inside the TGS-II was removed for this measurement. We found that the internal delay generator of the camera had to be set to about $13.7 \mathrm{~ns}$ additional delay, to optimize the recorded Rayleigh scattered signal. This number corresponds to our expectation. The time jitter in the triggering of the camera could not be measured exactly, but it was found to be less than 50 ps.

From Rayleigh scattered images with both slits removed from the TGS-II, we could also derive that after focusing, the FWHM diameter of the laser beam was roughly $150 \mu \mathrm{m}$. As the effective focal width in the old setup was around $400 \mu \mathrm{m}$, the new width is a considerable improvement, which helped, in combination with the narrower entrance slit, to achieve a better signal-to-background ratio in TS experiments.

Measurements with the slits in place showed that the apparatus profile in the spectral direction for the complete detection system was $0.6 \mathrm{~nm}$ FWHM. This is more than suf-

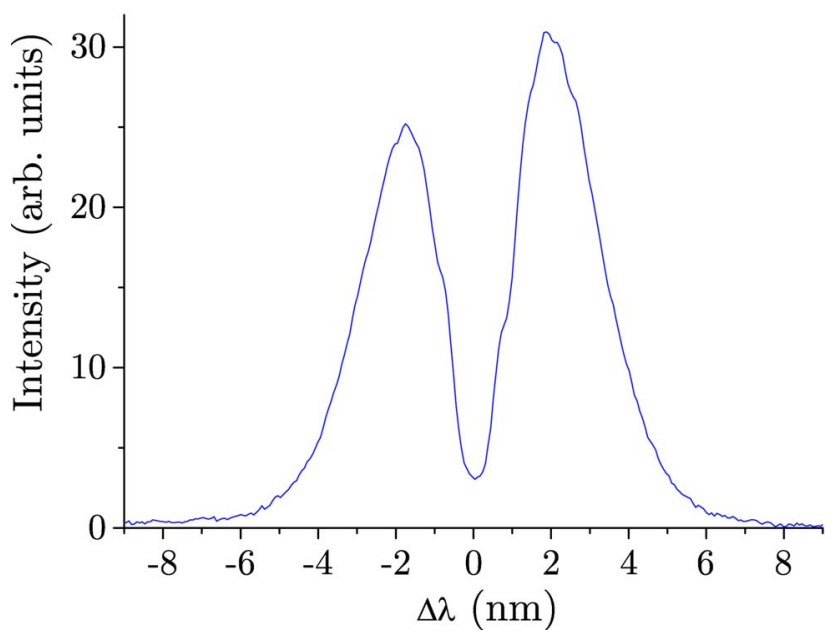

FIG. 4. Example of rotational Raman scattered spectrum from nitrogen gas. In this case, the signal was integrated over all spatial channels. 


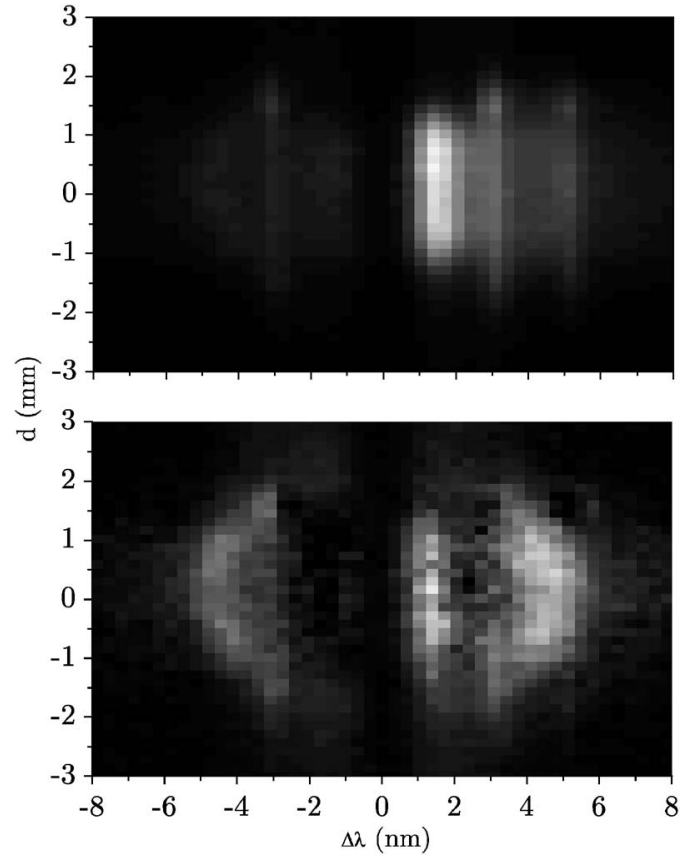

FIG. 5. Example of a Thomson scattered signal recorded with the new setup. Top frame: a "raw" vertically polarized signal, showing strong plasma emission in spectral lines. Bottom frame: the same signal, after subtraction of the horizontally polarized part of the plasma background. This image was seven times digitally intensified compared to the top image. The feature between $\Delta \lambda=1$ and $2 \mathrm{~nm}$ is a part of the plasma background that remained after background subtraction, but this part of the spectrum could simply be ignored while fitting the TS signal to a theoretical curve. The curved shapes between roughly 2 and $6 \mathrm{~nm}$ on either side of the spectrum represent the TS signal. The dark area in the center is due to the presence of the stray light filter.

ficient for Thomson scattering on EUV discharge plasmas. With this resolution, reasonably accurate results can be obtained as long as the electron temperature $T_{e}$ is larger than about $1.0 \mathrm{eV}$.

The suppression of stray light and Rayleigh scattered signal was verified by measuring the rotational Raman scattered signal from nitrogen gas. Raman scattering, being an inelastic scattering process, produces larger wavelength shifts from the laser wavelength than Rayleigh scattering does, but Rayleigh scattering has a much larger cross section. Therefore, observing a (rotational) Raman scattered spectrum without a strong Rayleigh and/or stray light peak in the center is a good test for the notch filter that has to block the central wavelength. Although the spectral resolution of the system-as given earlier-is not sufficient to resolve the individual Raman lines, the characteristic asymmetric shape of the overall spectrum could be clearly recognized; see Fig. 4 for an example.

In our TS experiments, we used a linear polarization filter to remove the plasma emission background from the TS signal. For this, we made use of the fact that polarization is preserved in the Thomson scattering process. Hence, in our geometry, with a vertically polarized laser beam, the Thomson scattered signal is also fully linearly polarized in the vertical direction. On the other hand, the plasma emission is in principle unpolarized. By use of the polarization filter, we could record the horizontally and vertically polarized com- ponents of the image on the camera separately. Now, when the horizontally polarized image is subtracted from the vertically polarized one, only the TS signal is ideally left in the result. This procedure makes polarization and wavelength dependent sensitivity measurements of the TGS-II necessary. Such measurements have been done with a ribbon lamp in the plasma position, and a rotatable linear polarization filter just in front of the second folding mirror. Later, the same filter was also used in TS experiments.

Our test experiments have shown that the new setup is suitable for Thomson scattering experiments. It can be used to study fast phenomena in plasmas in a spatially resolved way. When appropriate synchronization is available, up to $1 \mathrm{~ns}$ time resolution can be achieved if the plasma jitter is low enough. If the plasma setup can accept a trigger signal that comes less than about $60 \mathrm{~ns}$ in advance of the laser pulse (corresponding to the length of the delay line), a time resolution as low as 300 ps can even be achieved.

After the initial construction and characterization of the setup was finished, several series of TS experiments have been performed on the tin vapor discharge. An example of a recorded spatially resolved Thomson scattering signal is shown in Fig. 5. This particular signal was recorded during the decay phase of the tin vapor discharge, at about $400 \mathrm{~ns}$ after the pinch phase-see Ref. 8 for a description of the various discharge phases. The applied fitting procedure for the Thomson scattered signal can be found in Ref. 10. The electron density in the center of the plasma was found to be $1.7 \times 10^{23} \mathrm{~m}^{-3}$, while the electron temperature in the same position was about $3 \mathrm{eV}$, resulting in a fairly strongly collective Thomson scattering spectrum with scattering parameter $\alpha \approx 1.9$ - hence, the spectrum shows two broad peaks displaced from the central wavelength rather than a single Gaussian centered at $\Delta \lambda=0 \mathrm{~nm}$. With the conventional Thomson scattering setup, it would not have been possible to distinguish these features from the remaining plasma emission signal after background subtraction.

To allow for a detailed discussion of the discharge evolution and the related plasma physics, the experiments and an overview of their results will be presented in a separate publication.

${ }^{1}$ L. P. Bakker, J. M. Freriks, F. J. de Hoog, and G. M. W. Kroesen, Rev. Sci. Instrum. 71, 2007 (2000).

${ }^{2}$ A. Kono and K. Nakatami, Rev. Sci. Instrum. 71, 2716 (2000).

${ }^{3}$ M. J. van de Sande, Ph.D. thesis, Eindhoven University of Technology, 2002; http://alexandria.tue.nl/extra2/200210414.pdf.

${ }^{4}$ M. J. van de Sande and J. J. A. M. van der Mullen, J. Phys. D 35, 1381 (2002).

${ }^{5}$ M. J. van de Sande, R. H. M. Deckers, F. Lepkojus, W. Buscher, and J. J. A. M. van der Mullen, Plasma Sources Sci. Technol. 11, 466 (2002).

${ }^{6}$ X. Zhu, M. Redwitz, E. R. Kieft, M. J. van de Sande, and J. J. A. M. van der Mullen, J. Phys. D 37, 736 (2004).

${ }^{7}$ J. J. A. M. van der Mullen, G. Boidin, and M. J. van de Sande, Spectrochim. Acta, Part B 59, 929 (2004).

${ }^{8}$ E. R. Kieft, J. J. A. M. van der Mullen, G. M. W. Kroesen, V. Banine, and K. N. Koshelev, Phys. Rev. E 71, 026409 (2005).

${ }^{9}$ E. R. Kieft, J. J. A. M. van der Mullen, G. M. W. Kroesen, V. Banine, and K. N. Koshelev, Phys. Rev. E 70, 066402 (2004).

${ }^{10}$ E. R. Kieft, J. J. A. M. van der Mullen, G. M. W. Kroesen, V. Banine, and K. N. Koshelev, Phys. Rev. E 70, 056413 (2004).

${ }^{11}$ A. L. Vitushkin and L. F. Vitushkin, Appl. Opt. 37, 162 (1998).

${ }^{12}$ P. Klövekorn and J. Munch, Appl. Opt. 37, 1903 (1998). 